\title{
Software testing and evaluation in large-scale scientific applications
}

\author{
Mo $M u$ \\ The Hong Kong University of Science \& Technology \\ Department of Mathematics, Clear Water Bay, Kowloon, Hong Kong. \\ Email: mamu@uxmail.ust.hk
}

\begin{abstract}
In this article, the practical aspects of software testing and assessment are discussed in the context of large-scale scientific applications. We present difficulties in testing these scientific software and compare differences with the familiar mathematical software.
\end{abstract}

\section{Keywords}

Mathematical software, scientific software, large-scale applications, testing and evaluation, software reliability

\section{INTRODUCTION}

For over two decades people have realized the importance of software testing and evaluation in the numerical software community. There are basically two types of numerical software. One is general-purpose and numerical algorithm oriented. These are often called mathematical software. Examples are LAPACK, ITPACK, SPARSEPACK, IMSL, ELLPACK, MATLAB and MATHEMATICA. They range from library subroutine collections to software systems, to problem solving environments. Usually, this software is designed for model problems and mature numerical algorithms. Their developers have backgrounds in numerical analysis and computer science. Due to the efforts of this community, great progress has been made in developing rich benchmarks of test problems and evaluation metrics and tools to ensure the quality of mathematical software. In contrast, the other type of numerical software is scientific and engineering oriented. They target specific, but large-scale and complicated computational problems. For historical and technical reasons, most of this software is developed by scientists in various application disciplines. As observed in (Pool, 1997), the numerical software community faces a new crisis due to lack of their participation in the development of application software. We address in 
this article the practical aspects of software testing and evaluation in large-scale scientific applications.

\section{SCIENTIFIC APPLICATION SOFTWARE}

Scientific application software is built upon mathematical software to certain extent, and also upon even lower level numerical utilities like the IEEE floating-point standards and the BLAS routines. The availability of those high quality building blocks greatly relieves application developers from the burden of algorithmic details for routine computations, and also helps improve the quality of the entire computational engine. The quality of building blocks does not, however, ensure the overall quality of the system. Also as noted in (Pool, 1997), the traditional delivery mechanism, the standalone library of quality software, is increasingly irrelevant in today's computing environment, which makes the issue of software quality more difficult than in the traditional sense.

The development process of scientific software is quite different from that of mathematical software. In the former case, the software development is often tied with the computation and application. During this process, mathematical models and numerical methods may change, which in turn leads to changes in software structures and data structures. For instance, from numerical experiments with the developed system one may find that the model needs modifications, or the computational efficiency needs improvement so that new algorithms should be employed. This may imply a change of discretization methods from finite differences to finite elements, or the change of solvers from direct methods to iterative methods. Some changes may have minor impact on the existing system. But, very often the changes may make the system more and more messy if it is impractical to give up the original software. The quality of this software is thus less likely to be good. Testing and assessing them is even harder.

Among all aspects of software quality, the most essential is reliability. This, however, is challenging for large-scale scientific software due to the complexity of the application problems and computational methods. Let us consider a typical time-dependent, nonlinear problem in real science. The corresponding algorithm usually has the following structure. First, the time integration requires a loop over time steps. On each time level, a nonlinear problem needs to be solved if an implicit scheme is used. If Newton's method is applied, this iteration corresponds to another loop. Further, at each iterative step a sparse linear system is solved to compute the Newton update. If one chooses to use an iterative linear solver, say CG or GMRES depending on whether the Jacobian is symmetric positive definite, then there is one more nested loop. Now, there are also various choices to make in the algorithm. For the spatial discretization, one has to choose an affordable spacing in order to assure adequate resolution. For the time loop, one has to determine the ratio of step size versus spacing for the purpose of stability. For loops like the Newton iteration and GMRES iteration, appropriate stopping criteria should be used to terminate the loops. These issues are already theoretically difficult enough for a real application with even textbook algorithms because most of the analysis, such as discretization error, stability condition, and iterative convergence, can only be carried out for simple model problems. The algorithms may also be modified from the original forms in order to cope with a complicated application. Therefore, standard analyses are also not directly applicable. The 
mutual effects of the nested loops make it more difficult to assess the overall accuracy, or even the correctness. After all, in most of practical cases it is uncertain whether there exists a solution to the mathematical problem due to lack of theoretical results for complicated PDEs.

Three approaches are commonly used to test software reliability when there are no true solutions available for comparison. One is to compare computational results with experimental results. There exist uncertainties due to measurement errors in experiments and differences between models and real science. The second approach is to compare results computed by different software. Sometimes, different research groups report on different results and nobody knows which is reliable, or few existing results are available if a field is relatively new. The third testing approach is to compare results generated from running the software by varying certain parameter values to check the consistency of the results. This is, however, impractical for many very large-scale grand challenge applications because each run requires considerable time. In addition, results with low resolution often do not reveal true physical phenomena, and may have drastic differences from high-resolution results.

\section{SUMMARY}

We have presented the difficulties of software testing and assessment in large-scale scientific applications. These are lessons which we learned from our research project on superconductor simulation with parallel computing (Mu, 1996; Mu, Deng and Chou, 1996). We believe that they are common in other application fields. Great efforts are thus required to meet the challenge of software testing and evaluation in today's multidisciplinary scientific computing community.

\section{ACKNOWLEDGEMENTS}

This work was supported in part by RGC HKUST593/94E.

\section{REFERENCES}

Mu, M. (1996) A linearized Crank-Nicolson-Galerkin method for the Ginzberg-Landau model. SIAM J. Sci. Comput., to appear.

$\mathrm{Mu}, \mathrm{M}$. (1996) Superconductor simulation with parallel computing, in preparation.

Mu, M., Deng, Y. and Chou, C. (1996) Numerical methods for simulating GinzburgLandau vortices. SIAM J. Sci. Comput., to appear.

Pool, J. (1997) Is numerical software relevant? Is it too late to worry about quality? in Quality of Numerical software: Assessment and Enhancement (ed. R. F. Boisvert), Chapman \& Hall, London. (this volume) 